Typeset using jjaptex.sty <ver.2.1.2>

\title{
Relationship between Control of Reactive Plasmas with Magnetic Filter and Formation of Thin Films
}

Osamu Fukumasa, Yasushi Tauchi and Satoshi SakiYama

Department of Electrical and Electronic Engineering, Yamaguchi University, Tokiwadai 2557, Ube 755, Japan

(Received

Plasma parameters $\left(\mathrm{CH}_{4} / \mathrm{H}_{2}+\right.$ Ar plasmas $)$ are spatially well controlled using a movable magnetic filter. At any filter position, plasma parameters change dramatically across the magnetic filter. The plasma is divided into two parts, the source plasma region (high density plasmas with energetic electrons) and the diffused plasma region (low electrontemperature plasmas without energetic electrons). Carbon thin films are prepared well in the diffused plasma region. The effects of bias potential of the substrate and control of neutral radicals on formation of thin films are discussed briefly.

KEYWORDS: plasma CVD, magnetic filter, electron energy distribution function, multi cusp plasma source, spatial control, reactive plasmas 


\section{Introduction}

The plasma chemical vapor deposition (p-CVD) method has been used successfully in the preparation of various kinds of thin films. To this end, plasma parameters, especially the energy distribution and density of electrons, must be carefully controlled, because, in the initial processes, decomposition of source gas molecules or production of reactive species including plasma production, are triggered by electron collisions.

To date, we have studied the applicability of a magnetically filtered multicusp plasma source to a tandem p-CVD reactor. ${ }^{1)}$ We have shown that the addition of a movable magnetic filter and a plasma grid can spatially control the electron energy distribution function (EEDF) and plasma parameters in reactive plasmas. ${ }^{2,3)}$ In this paper, we will further discuss the control of plasma parameters for various operating conditions and the relationship between the control of plasma parameters and the formation of thin films.

\section{Experimental Setup and Procedure}

Figure 1 shows a schematic of the apparatus. The details are reported elsewhere. ${ }^{2,3)}$ The source chamber $(16 \mathrm{~cm}$ diameter, $30 \mathrm{~cm}$ long) is made of stainless steel and the outside wall of the chamber is lined with 8 rows of ferrite permanent magnets arranged in an alternating north pole-south pole geometry. This surface magnetic field layer confines primary electrons and produced plasmas well.

Gas mixtures of $\left(\mathrm{H}_{2}+\mathrm{Ar}\right)$ and $\mathrm{CH}_{4}$ were continuously introduced into the source chamber. Steady-state plasmas were produced by dc discharge between the tungsten filaments cathode and the chamber anode with discharge voltage $V_{\mathbf{d}}$ and discharge current $I_{\mathrm{d}}$. Plasma parameters were measured by two cylindrical Langmuir probes $(1.0 \mathrm{~mm}$ diameter, $3 \mathrm{~mm}$ long). To obtain the EEDF using the Druyvesteyn method, the second derivative of the probe characteristics was also measured. Information on neutral particles was obtained with emission spectroscopy.

A samarium-cobalt magnetic filter divides the entire chamber into an arc discharge (source region, region I) and a diffused plasma (deposition region, region II). This magnetic filter composed of two rods provides a limited region of transverse magnetic field (an integrated magnetic field of about $130 \mathrm{G} \cdot \mathrm{cm}$ ) where only electrons are magnetized. The 
magnetic field is strong enough to prevent all energetic primary electrons in region I from entering into region II. However, cold electrons, together with positive ions, can penetrate the filter and form a diffused plasma.

\section{Experimental Results and Discussion}

\subsection{Effect of the magnetic filter on controlling plasma parameters}

According to previous results, ${ }^{1-3}$ ) a remarkable change in plasma parameters (such as EEDF, electron density $n_{\mathrm{e}}$, electron temperature $T_{\mathrm{e}}$ and floating potential $V_{\mathrm{f}}$.) across the magnetic filter is clearly observed. Even if $\mathrm{CH}_{4}$ gas is introduced into $\mathrm{H}_{2}+\mathrm{Ar}$ plasmas, the magnetic filter can spatially control the EEDF and the related plasma parameters. Here, we continue on discussion of these problems, including the effects of the gas flow rate and total gas pressure $p$ on the production and control of these plasmas.

Figure 2 shows the spatial variations of $n_{\mathrm{e}}$ and $T_{\mathrm{e}}$ in $\mathrm{H}_{2}+\mathrm{Ar}$ plasmas for three different flow rates under a constant $p=2 \mathrm{mTorr}$, where the magnetic filter position $L_{\mathrm{f}}=17 \mathrm{~cm}$ apart from the plasma mesh grid. In Fig. 2 , the magnetic filter position $\left(L_{\mathbf{f}}=17 \mathrm{~cm}\right)$ corresponds to $L=0$, and the source and the diffused plasma regions are on the right and the left sides, respectively. Partial pressures of $\mathrm{H}_{2}$ and $\mathrm{Ar}$ gases are $1 \mathrm{mTorr}$ and $1 \mathrm{mTorr}$, respectively. Both $n_{\mathrm{e}}$ and $T_{\mathrm{e}}$ vary markedly in their magnitudes across the filter. However, the spatial distributions of $n_{\mathrm{e}}$ and $T_{\mathrm{e}}$ have similar patterns in spite of the different gas flow rates. Namely, plasma parameters have almost the same magnitudes for various gas flow rates if $p$ is kept at a certain constant value.

Figure 3 shows the spatial variation of $n_{\mathrm{e}}$ and $T_{\mathrm{e}}$ for three different pressures under constant gas flow rate $\left(\mathrm{H}_{2} / \mathrm{Ar}=32 / 9.9 \mathrm{sccm}\right)$. With increasing $p, n_{\mathrm{e}}$ and $T_{\mathrm{e}}$ vary in magnitude although their spatial dependence on the filter effect appears to be similar.

As shown previously, ${ }^{1-3)}$ the magnetic filter prevents only the high-energy electrons from entering into region II. This feature of the magnetic filter is the most important point for controlling the EEDF. Figure 4 shows spatial variations of $V_{\mathrm{f}}$ corresponding to the results in Fig. 2. Apparently, $V_{\mathrm{f}}$ changes markedly across the filter. This shows another example of the effect of the magnetic filter as $V_{\mathrm{f}}$ depends on the high energy tail of the EEDF. Controlling $V_{\mathrm{f}}$ is important in etching plasmas when the energy of the etching ions 
is directly related to $V_{\mathrm{f}}$.

According to the results shown in Figs 2-4, it is reconfirmed ${ }^{3)}$ that plasma parameters $\left(n_{\mathrm{e}}, T_{\mathrm{e}}, V_{\mathrm{f}}\right)$ for various operating conditions change dramatically across the magnetic filter, and that the plasma is divided into the distinct regions of the source plasma with highenergy electrons and high $T_{\mathrm{e}}$ (region I) and the diffused plasma without high-energy electrons and with low $T_{\mathrm{e}}$ (region II).

On this preferential reflection of high-energy electrons at the magnetic filter, as explained by Coulomb collisions in the presence of a magnetic field was previously reported. ${ }^{4)}$ However, this is not the case in the present experiment and the role of the magnetic filter is not well clarified. Therefore, we have proposed the following model ${ }^{2,3)}$ for the preferential reflection of high-energy electrons. Electrons entering the magnetic filter are trapped in filter field $\boldsymbol{B}$. Then, most electrons cross the magnetic filter due to $\boldsymbol{E} \times \boldsymbol{B}$ drift where $\boldsymbol{E}$ represents thermally excited low frequency electrostatic fluctuations. Therefore, $\boldsymbol{E} \times \boldsymbol{B}$ drift for electrons decreases with the increase of electron velocity (i.e., energy), because fluctuating electric fields are averaged over their finite Larmor radii. Namely, high-energy electrons diffuse at a lower rate than do low-energy electrons. Both simulation results ${ }^{3)}$ and experimental results ${ }^{2)}$ support the model of the magnetic filter described above.

For control of $T_{\mathrm{e}}$ in weakly ionized plasmas, a grid-bias method has been proposed by Kato et al. ${ }^{5)}$ Control of $T_{\mathrm{e}}$ is performed on plasmas passing through a coarse mesh grid from a discharge region. By increasing the negative potential applied to the grid (from $-5 \mathrm{~V}$ to $-15 \mathrm{~V}), T_{\mathrm{e}}$ is continuously decreased from $1.3 \mathrm{eV}$ to $0.035 \mathrm{eV}$. In future study, we will examine the relationship between the magnetic filter method and the grid-bias method.

The multicusp plasma sources with a magnetic filter are widely used and proposed as promising sources of high current $\mathrm{H}^{-} / \mathrm{D}^{-}$ions for NBI heating in the fusion community. ${ }^{6,7)}$ $\mathrm{H}^{-}$ions are generated by the dissociative attachment of slow plasma electrons $\left(T_{\mathrm{e}} \sim\right.$ $1 \mathrm{eV})$ to highly vibrationally excited hydrogen molecules $\mathrm{H}_{2}(v ")$, where these $\mathrm{H}_{2}\left(v^{\prime \prime}\right)$ are primarily produced by the collisional excitation of fast electrons with energies in excess of $20-30 \mathrm{eV}$. $)$ With the use of the magnetic filter, the EEDF is optimized for the two-step process of $\mathrm{H}^{-} / \mathrm{D}^{-}$formation. Therefore, these types of plasma sources are available for 
the production of negative ion plasmas, such as $\mathrm{Cl}^{-}, \mathrm{O}^{-}$and $\mathrm{SF}_{6}{ }^{-}$.

\subsection{Preparation of thin films}

To date, we have successfully confirmed that the plasma parameters are spatially well controlled using the movable magnetic filter. Next, in order to clarify the relationship between the preparation of thin films and the control of plasmas, thin films are prepared in both the source and the diffused plasma regions. Carbon thin films are prepared on Mo substrates as functions of the gas flow rate and the substrate potential by supplying $\mathrm{CH}_{4}$ gas to $\mathrm{H}_{2}+\mathrm{Ar}$ plasma. Properties of deposited thin films were analyzed with X-ray diffraction, Raman spectroscopy, scanning electron microscopy (SEM) and infrared (IR) absorption spectroscopy methods.

For a typical example of thin film formation, under the conditions whereby the material gas flow rate $\mathrm{H}_{2} / \mathrm{Ar} / \mathrm{CH}_{4}$ is $32 / 10 / 4 \mathrm{sccm}$ and deposition time is $6 \mathrm{~h}$, thin films are prepared in both plasma regions. Figure 5 shows SEM photographs of the prepared thin films, where the substrate potential is the earth potential and the substrate position $L_{\text {sub }}$ is $15 \mathrm{~cm}$ away from the plasma grid. The film in Fig. 5(a) is deposited in the source region, where $p=2.3 \mathrm{~m}$ Torr and the magnetic filter position $L_{\mathrm{f}}$ is $13 \mathrm{~cm}$ away from the plasma grid. The films in Fig. 5(b) and Fig. 5(c) are deposited in the diffused plasma region, where $L_{\mathrm{f}}=17 \mathrm{~cm}$ and $p=2.3 \mathrm{mTorr}$ in Fig. $5(\mathrm{~b})$ and $4.6 \mathrm{mTorr}$ in Fig. $5(\mathrm{c})$.

As is shown in Fig. 2, under constant gas pressure, plasma parameters are maintained at almost constant levels for the different gas flow rates. However, film formation (especially in the diffused plasma region) depends strongly on the gas flow rate. With gas flow rates decreasing to $9 / 2 / 0.6 \mathrm{sccm}(p=2.3 \mathrm{mTorr}$, see Fig. $5(\mathrm{~b}))$, thin films are not prepared in the diffused plasma region, even if the deposition time is 6 or $8 \mathrm{~h}$ (Case A). However, thin films are prepared in about one hour when the gas flow rate $\mathrm{H}_{2} / \mathrm{Ar} / \mathrm{CH}_{4}$ is $32 / 10 / 4 \mathrm{sccm}$ (Case B). Deposition time $(1 \mathrm{~h})$ in Case B is shorter by a factor 6 or 8 than that $(6$ or $8 \mathrm{~h}$ ) in Case $\mathrm{A}$. On the other hand, the gas flow rate of $\mathrm{CH}_{4}$ in Case $\mathrm{B}$ is $6-7$ times larger than that in Case A. Then, during the deposition time, the accumulated particle flux of $\mathrm{CH}_{\mathrm{x}}$ to the substrate may be almost the same for the two cases although film formation is quite different. Therefore, these two results suggest that gas flow rate not only affects 
radical transport but also enhances film formation (i.e., surface reaction). This point is not clear at this time.

We have also confirmed the following characteristic features of film formation. In the diffused plasma region, two types of thin films are prepared depending upon whether the substrate potential is the floating or earth potential. On the other hand, films prepared in the source region are of only one type, regardless of the substrate potential.

In both regions, the films deposited with earth potential (type I) have the Raman shift such as shown in Fig. 6. Therefore, the films prepared in the diffused plasma region have the same characteristics as those in the source region. According to the $\mathrm{X}$-ray diffraction patterns, there are no distinguishable peaks except those of the substrate material. So, the thin films prepared in both plasma regions may be amorphous carbon although detailed characterization of film quality is currently under study.

Under the condition that the substrate potential is floating $(-3 \sim-6 \mathrm{~V})$, a second type of thin film (type II) is deposited in the diffused plasma region, although only type I films are prepared in the source plasma region, where the substrate potential is about $-30 \mathrm{~V}$. Thin films in the diffused plasma region exhibit the IR spectrum shown in Fig. 7. The absorptions derived from the bonds between $\mathrm{C}$ and $\mathrm{H}$ are observed. In the IR spectrum of the type I films shown in Fig. 5, these absorptions are not observed. The effect of substrate potential is a topic for further study. In the diffused plasma region, thin films are prepared for two different substrate potentials, i.e., $+10 \mathrm{~V}$ and $-10 \mathrm{~V}$. When the substrate potential is set at $-10 \mathrm{~V}$, prepared films are the same as the type II film. On the other hand, when the substrate potential is set at $+10 \mathrm{~V}$, prepared films are the same as the type I film. From these results, it can be concluded that thin films prepared in the diffused plasma region are well controlled by varying the substrate potential.

In addition to the above experimental results, plasma parameters in the diffused plasma regions are easily controlled by changing the field strength of the magnetic filter and the potential of the plasma grid. The diffused plasma region of this plasma source should be a good reactor for precisely processing materials. 


\section{Conclusions}

We have studied the potential for control of reactive plasmas with the movable magnetic filter, and the applicability of the plasma source with the magnetic filter to p-CVD. Spatial control of plasma parameters with a magnetic filter is well established. This method of plasma parameter control can also be applied to plasmas produced by radio-frequency and electron cyclotron resonance discharges. Preparation of thin films is realized in the diffused plasma region. Furthermore, thin film formation in this region is well controlled by varying substrate potential and gas flow rate. Therefore, this plasma source could be applied to processing plasmas to determine the optimum conditions for material processing.

In the future, we will attempt to control plasma parameters continuously by varying the field intensity of the magnetic filter. Characterization of prepared thin films and clarifying the relationship between the control of plasma parameters and thin film formation are also ongoing investigations. 


\section{References}

1) O. Fukumasa, H. Naitou and S. Sakiyama: Jpn. J. Appl. Phys. 30 (1991) L1063.

2) O. Fukumasa, H. Naitou and S. Sakiyama: T. IEE Jpn. 111-A (1991) 1057.

3) O. Fukumasa, H. Naitou and S. Sakiyama: J. Appl. Phys. 74 (1993) 848.

4) A. J. T. Holmes: Rev. Sci. Instrum. 53 (1982) 1517.

5) K. Kato, S. Iizuka and N. Sato: Appl. Phys. Lett. 65 (1994) 816.

6) See for example Proc.7th Int. Symp. on the Production and Neutralization of Negative Ions and Beams (Brookhaven, 1995)(AIP Conf. Proc. 380).

7) O. Fukumasa: Shinkuu 38 (1995) 903. (in Japanese)

8) O. Fukumasa: J. Appl. Phys. 71 (1992) 3193. 


\section{Figure captions}

Fig. 1. Schematic of the multicusp plasma source equipped with the movable magnetic filter.

Fig. 2. Axial variation of $n_{\mathrm{e}}(\mathrm{a})$ and $T_{\mathrm{e}}(\mathrm{b})$ for three different gas flow rates in $\mathrm{H}_{2}+\mathrm{Ar}$ plasma, where the magnetic filter position $\left(L_{\mathrm{f}}=17 \mathrm{~cm}\right)$ corresponds to $L=0$. Experimental conditions are as follows: discharge voltage $V_{\mathrm{d}}=80 \mathrm{~V}$ and current $I_{\mathrm{d}}=2 \mathrm{~A}$. Total pressure is maintained at $2 \mathrm{~m}$ Torr.

Fig. 3. Axial variation of $n_{\mathrm{e}}(\mathrm{a})$ and $T_{\mathrm{e}}$ (b) for three different total gas pressures $p(2,3$, $4 \mathrm{mTorr})$, where the filter position $\left(L_{\mathrm{f}}=17 \mathrm{~cm}\right)$ corresponds to $L=0$. Gas flow rates of $\mathrm{H}_{2}$ and $\mathrm{Ar}$ are maintained at 32 and $9.9 \mathrm{sccm}$, respectively.

Fig. 4. Axial variation of $V_{\mathrm{f}}$ corresponding to the results in Fig. 2.

Fig. 5. SEM images of the prepared thin films for two different regions: (a) source plasma region $\left(L_{\mathrm{f}}=13 \mathrm{~cm}\right)$, (b) and $(\mathrm{c})$ diffused plasma region $\left(L_{\mathrm{f}}=17 \mathrm{~cm}\right)$. Experimental conditions are as follows: $V_{\mathrm{d}}=80 \mathrm{~V}, I_{d}=2 \mathrm{~A}, p=2.3 \mathrm{~m}$ Torr for (a) and (b), and 4.6 mTorr for (c). Gas flow rate of $\mathrm{H}_{2} / \mathrm{Ar} / \mathrm{CH}_{4}=32 / 10 / 4 \mathrm{sccm}$. Substrate potential is the earth potential and substrate position $L_{\mathrm{sub}}=15 \mathrm{~cm}$.

Fig. 6. Raman Spectra of the prepared thin films (type I), corresponding to the results shown in Figs. 5(b) and 5(c), where the substrate potential is the earth potential.

Fig. 7. IR spectrum of the prepared thin film (type II), where the substrate potential is the floating potential. Experimental conditions are the same as in Fig. 5 except that the substrate potential is the floating potential $(-4.5 \mathrm{~V})$. 


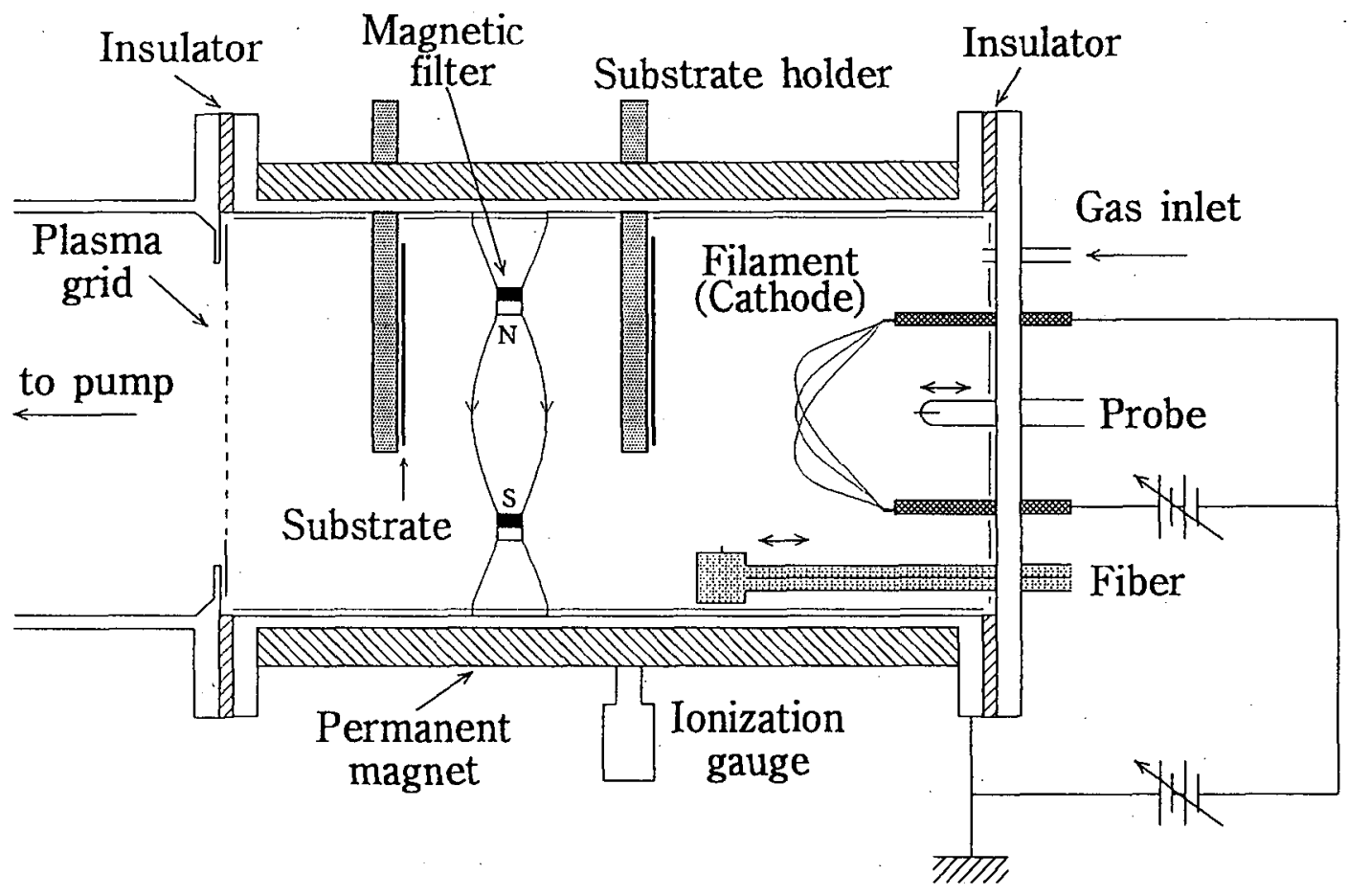

Fig.1 

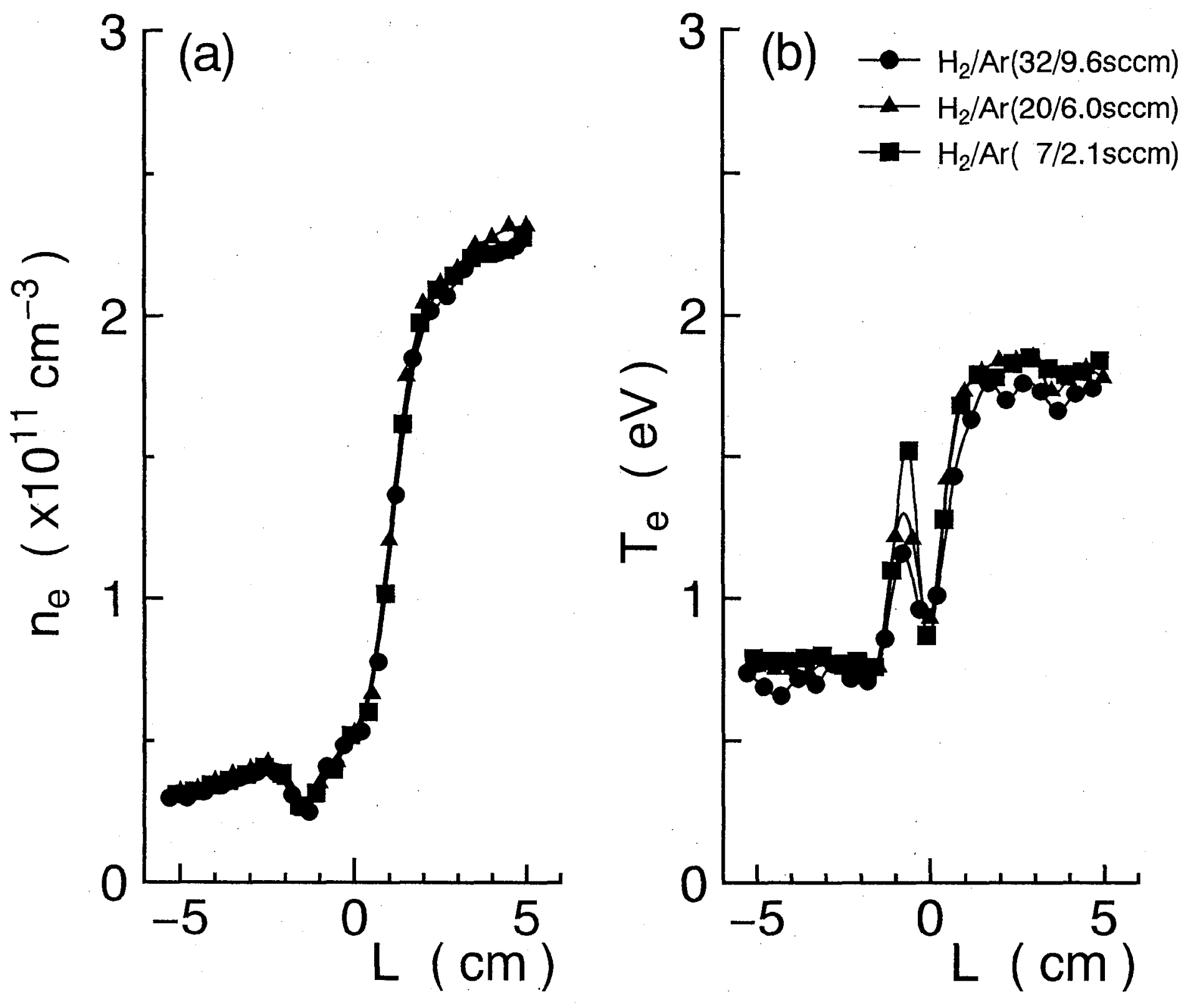

Fig.2 

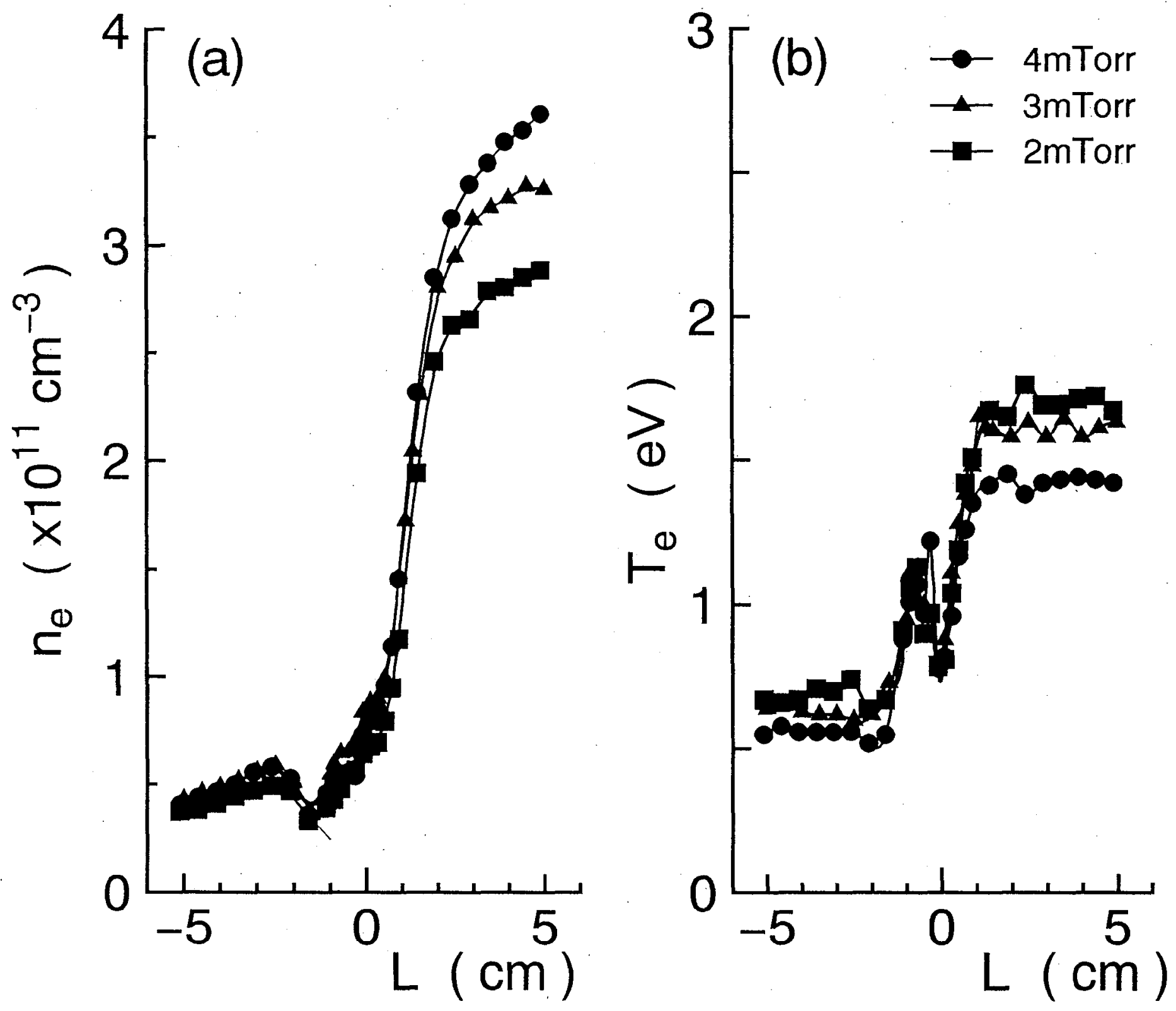

Fig.3 


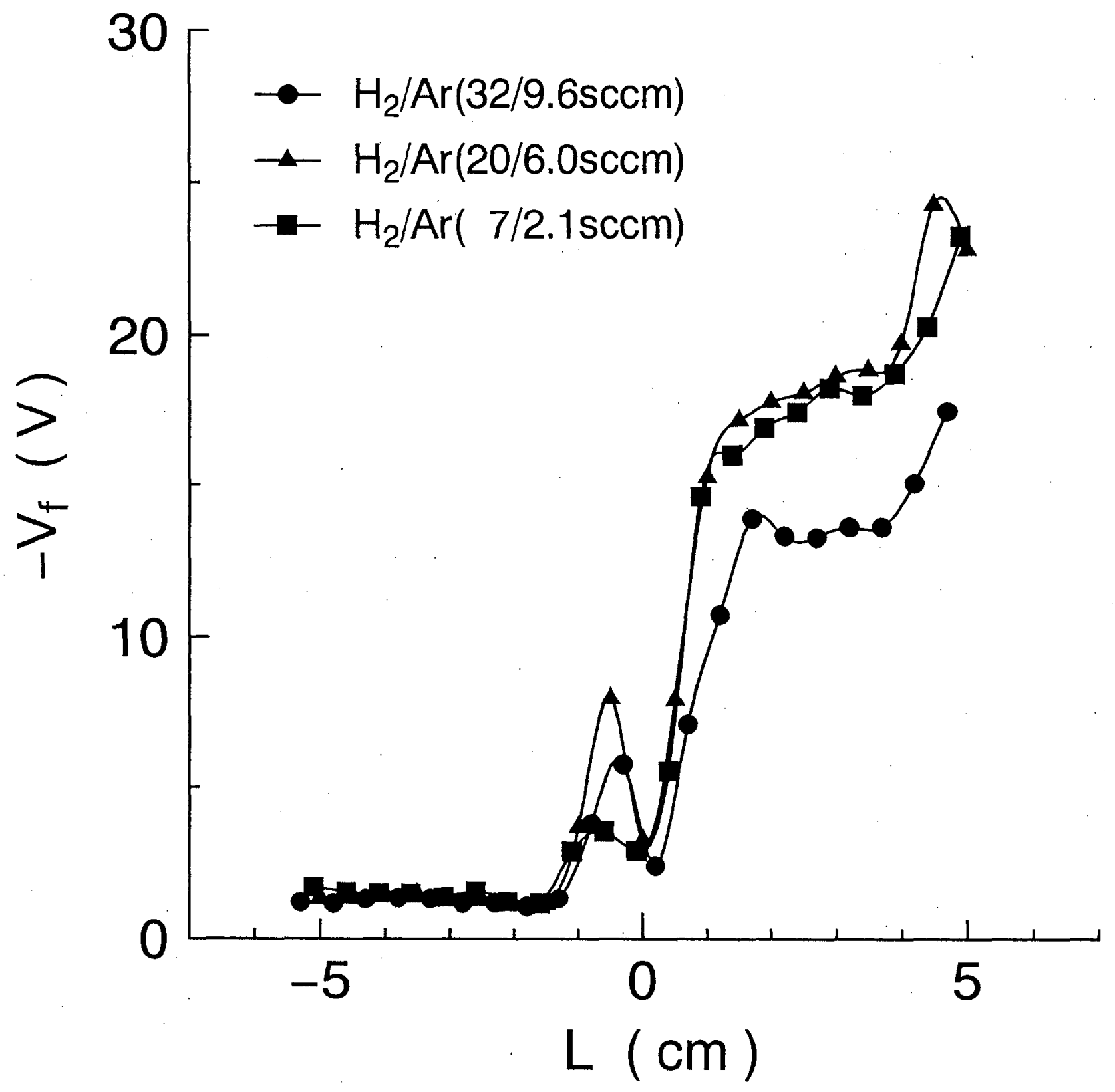

Fig.4 
(a)

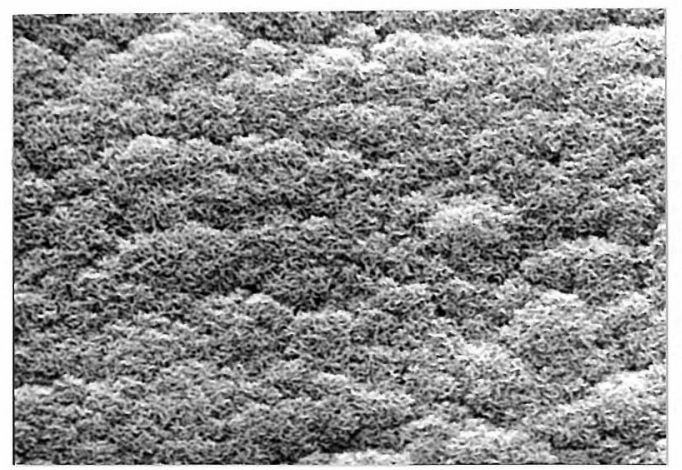

ل $5 \mu \mathrm{m}$ (b)

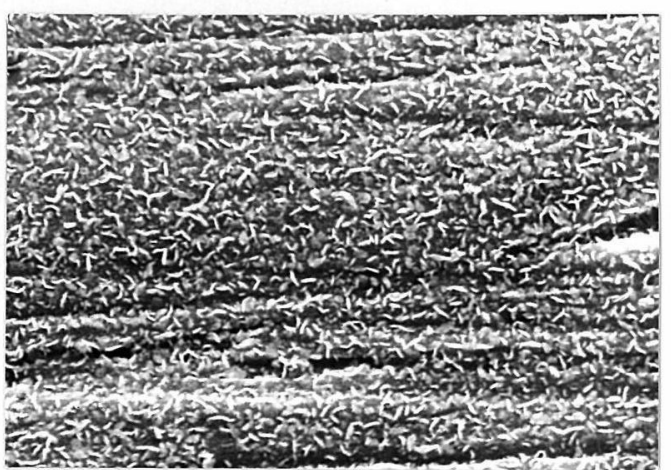

$5 \mu \mathrm{m}$ (c)

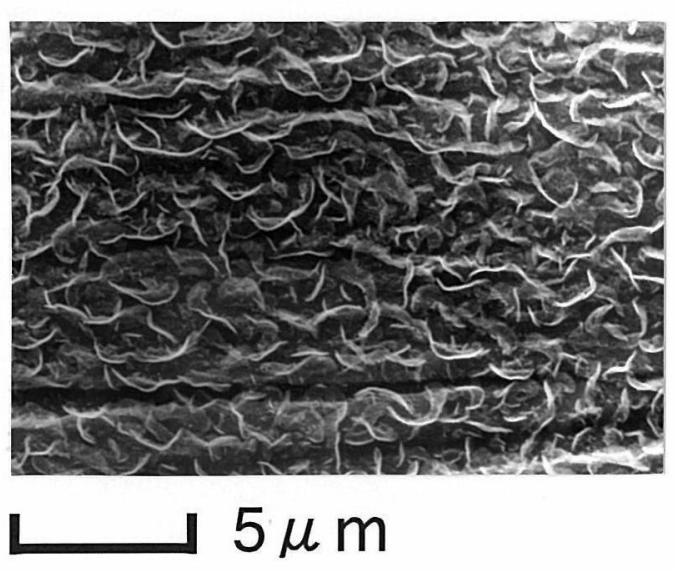

Fig.5 


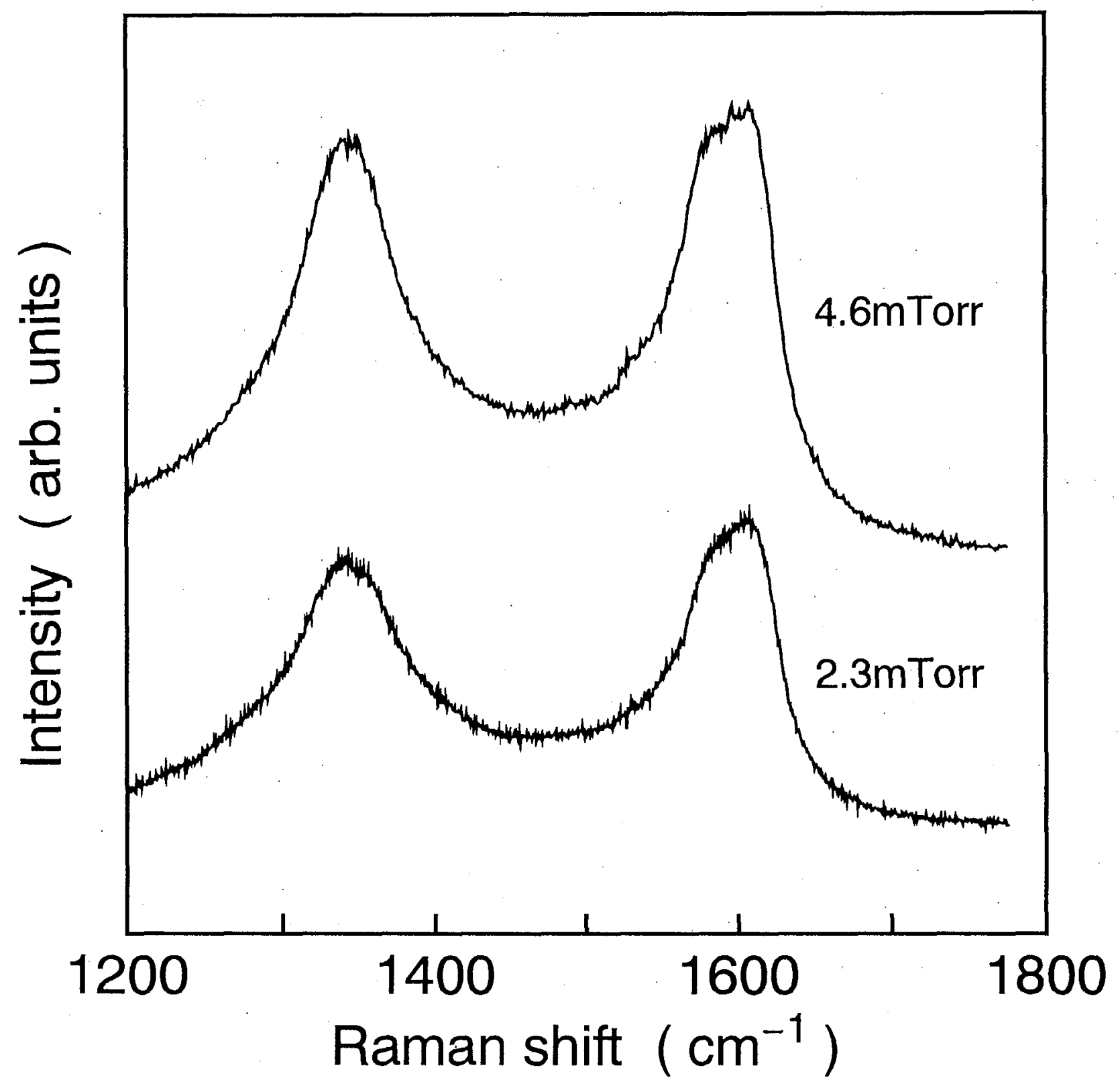

Fig.6 


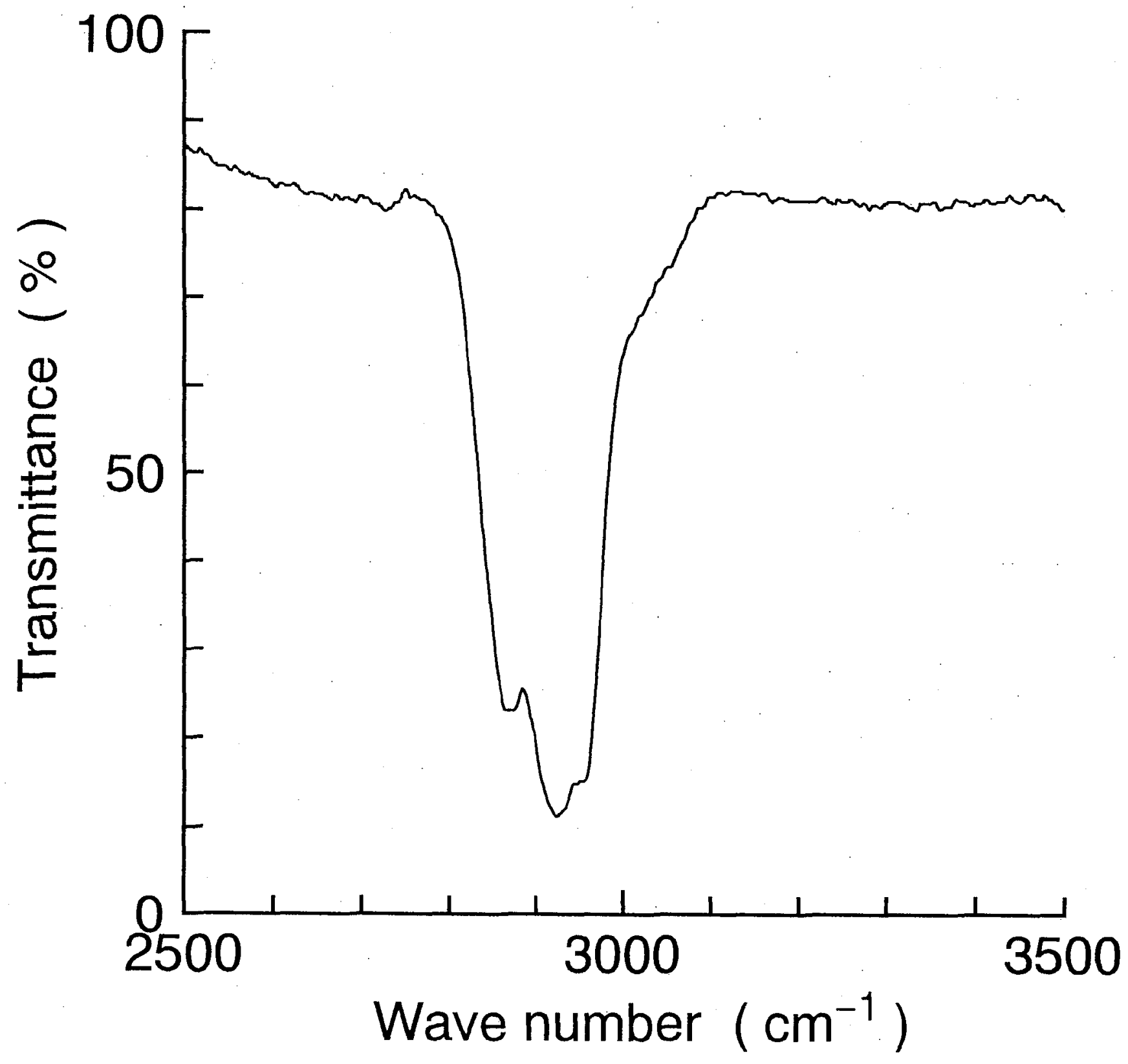

Fig.7 